\title{
Influence of van der Waals forces on the adsorption structure of benzene on silicon studied using density functional theory
}

Johnston, Karen; Kleis, Jesper; Lundqvist, Bengt; Nieminen, Risto M.

Published in:

Physical Review B Condensed Matter

Link to article, DOI:

10.1103/PhysRevB.77.121404

Publication date:

2008

Document Version

Publisher's PDF, also known as Version of record

Link back to DTU Orbit

Citation (APA):

Johnston, K., Kleis, J., Lundqvist, B., \& Nieminen, R. M. (2008). Influence of van der Waals forces on the adsorption structure of benzene on silicon studied using density functional theory. Physical Review $B$ Condensed Matter, 77(12), 121404. https://doi.org/10.1103/PhysRevB.77.121404

\section{General rights}

Copyright and moral rights for the publications made accessible in the public portal are retained by the authors and/or other copyright owners and it is a condition of accessing publications that users recognise and abide by the legal requirements associated with these rights.

- Users may download and print one copy of any publication from the public portal for the purpose of private study or research.

- You may not further distribute the material or use it for any profit-making activity or commercial gain

- You may freely distribute the URL identifying the publication in the public portal 


\title{
Influence of van der Waals forces on the adsorption structure of benzene on silicon studied using density functional theory
}

\author{
Karen Johnston, ${ }^{1}$ Jesper Kleis, ${ }^{2}$ Bengt I. Lundqvist, ${ }^{2,3}$ and Risto M. Nieminen ${ }^{1}$ \\ ${ }^{1}$ Laboratory of Physics, Helsinki University of Technology, P.O. Box 1100, FIN-02015 HUT, Finland \\ ${ }^{2}$ Center for Atomic-Scale Materials Design, Technical University of Denmark, DK-2800 Kongens Lyngby, Denmark \\ ${ }^{3}$ Department of Applied Physics, Chalmers University of Technology, Gothenburg SE-41296, Sweden
}

(Received 20 February 2008; published 27 March 2008)

\begin{abstract}
Two different adsorption configurations of benzene on the $\mathrm{Si}(001)-(2 \times 1)$ surface, the tight-bridge and butterfly structures, were studied using density functional theory. Several exchange and correlation functionals were used, including the recently developed van der Waals density functional (vdW-DF), which accounts for the effect of van der Waals forces. In contrast to the Perdew-Burke-Ernzerhof (PBE), revPBE, and other generalized-gradient approximation functionals, the vdW-DF finds that, for most coverages, the adsorption energy of the butterfly structure is greater than that of the tight-bridge structure.
\end{abstract}

DOI: 10.1103/PhysRevB.77.121404

PACS number(s): 68.43.Bc, 31.15.es, 34.35. $+\mathrm{a}, 71.15 . \mathrm{Mb}$

In the quest for reduced-sized transistor chips, the combination of organic molecules and silicon-based technology is of increasing importance. The ability to manipulate organic molecules on surfaces is developing rapidly and an understanding of the structural and transport properties of adsorbed molecules is essential. ${ }^{1-5}$

Silicon is not only important for technology but also demonstrates the versatility of the covalent bond. Covalency makes bulk silicon, diamond, and graphene layers very strong. At the same time, it can produce a multitude of competing atomic structures when spatial restrictions are released. For instance, the $\mathrm{Si}(111)$ and $\mathrm{Si}(001)$ surfaces show quite different properties, including radically different types of reconstructions. While the covalent bond is typically very strong, the energy differences between such reconstructions can be small. ${ }^{6}$ Their relative stabilities and the influence from adsorbates are interesting issues to understand. In particular, there may be situations where the weak van der Waals (vdW) force can significantly influence the structures. The benzene molecule demonstrates several types of internal bonds, typically interacts with other molecules via vdW forces, and is an important model unit for several classes of large molecules such as DNA. ${ }^{7}$ The adsorption of benzene on $\mathrm{Si}$ is obviously very interesting because of both the versatility in bond types and the wide ramifications, including technological ones, such as molecular electronics.

Adsorption of benzene on the $\operatorname{Si}(001)-(2 \times 1)$ surface has been studied extensively but without unanimous results. There is agreement on the two most stable structures (shown in Fig. 1), but to date, there is no agreement on which is the stable one. The butterfly (BF) structure is adsorbed on top of a single dimer and has two symmetry planes: along and perpendicular to the dimer row. The tight-bridge (TB) structure is adsorbed across two dimers and has one symmetry plane along the dimer row.

To differentiate between the adsorption structures and their bonding and symmetry properties, a variety of experimental tools have been employed. Thermal desorption and angle-resolved photoelectron spectroscopy were used to investigate the electronic structure and symmetry of benzene on $\mathrm{Si}^{8}$ and a single dimer structure was observed, supporting the BF configuration. Near-edge $\mathrm{x}$-ray-absorption fine structure found the benzene to be symmetric with respect to the dimer axis, ruling out the TB structure. ${ }^{9}$ These findings were supported by optical spectroscopy data, ${ }^{10}$ where it was found that benzene adsorbs on top of a single dimer rather than on the bridge site between two dimers. However, according to scanning tunneling microscopy (STM) studies, ${ }^{11,12}$ the benzene molecule adsorbs initially in the BF structure but this is observed to be metastable with respect to a bridging configuration. With the STM tip, one benzene structure can be converted to another, with the conversion-energy barrier estimated to be $0.95 \mathrm{eV}$. A high-resolution study ${ }^{13}$ suggested that the adsorption geometry depends on coverage ${ }^{37}$ and showed that a bridging structure is favored at low coverages, while at high coverages, a single dimer structure $(\mathrm{BF})$ is more stable. This would explain the discrepancy between the experimental results of Refs. 8-10 that supported the BF structure, as they were performed at saturation coverage, while the STM experiments were carried out at a low coverage of $0.044 \mathrm{ML}$.

Adsorption energies $E_{\text {ads }}$ have been calculated using standard density functional theory (DFT) and all of these studies $^{14-16}$ support the TB structure. An MP2 (second order
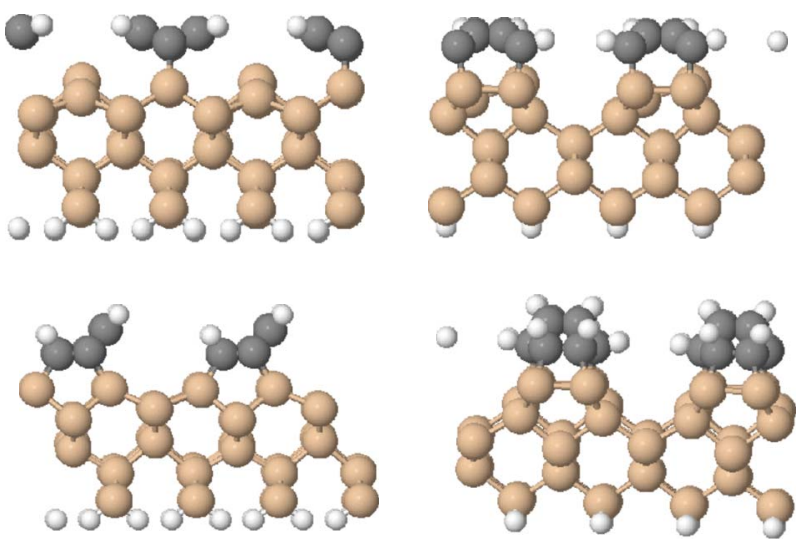

FIG. 1. (Color online) BF (top) and TB (bottom) structures of $\mathrm{C}_{6} \mathrm{H}_{6}$ on $\mathrm{Si}(001)-(2 \times 1)$. Left hand side: viewed along [110]. Right hand side: viewed along [110] dimer rows. 
TABLE I. $E_{\text {ads }}$ of benzene in the TB and BF geometries for a coverage of $0.5 \mathrm{ML}$. The pseudopotentials and $\mathrm{xc}$ functionals used are also shown.

\begin{tabular}{lcccc}
\hline \hline \multicolumn{2}{c}{$E_{\text {ads }}(\mathrm{eV} / \mathrm{molecule})$} & & & \\
\cline { 1 - 2 } TB & BF & Pseudopotential & GGA & Reference \\
\hline 0.66 & 0.47 & US & revPBE & Present \\
1.19 & 0.92 & US & PBE & Present \\
1.24 & 0.99 & US & PW91 & Present \\
1.25 & 1.00 & PAW & PW91 & 32 \\
1.05 & 0.82 & US/NC & PBE & 15 \\
\hline \hline
\end{tabular}

perturbation/quantum mechanics molecular dynamics) cluster calculation ${ }^{17}$ is the only calculation that supports the $\mathrm{BF}$ structure $\left(E_{\mathrm{ads}}=1.04 \mathrm{eV}\right)$. Unlike standard DFT calculations, MP2 methods include vdW forces but because they are computationally heavy, only small systems can be treated with this method. Si (001) is an extended material and it is not clear whether small clusters can give accurate adsorption energies. Furthermore, the cluster geometry corresponds to a low coverage situation, so it cannot be compared with saturation coverage results.

The vdW-DF ${ }^{18}$ was developed to account for the effect of $\mathrm{vdW}$ forces in DFT. It has been shown to give accurate results for molecular systems, such as benzene dimers, ${ }^{19,20}$ and recently, it has been successfully applied to systems with covalent bonding present. ${ }^{21-23}$ It is currently believed that $\mathrm{vdW}$ forces are only important in physisorbed systems but in this paper, we demonstrate that this is not the case. By using vdW-DF in DFT calculations, we show that in most of the studied configurations, vdW forces stabilize the BF structure, which is surprising since the TB structure has more covalent bonds and would be expected to be more strongly bound to the surface.

Standard DFT calculations were performed with DACAPO (Ref. 24) using the Perdew-Burke-Ernzerhof (PBE) form $^{25-27}$ of the generalized-gradient approximation (GGA) and ultrasoft (US) pseudopotentials. The plane-wave energy cutoff was $400 \mathrm{eV}$ and the Brillouin zone mesh used was equivalent to $4 \times 4 \times 1$ Monkhorst-Pack $k$-point sampling for $0.5 \mathrm{ML}$ coverages. We used nine-atomic layer $\mathrm{Si}$ slabs, $\approx 15 \AA$ of vacuum, and a lattice constant of $5.47 \AA$. To save computational time, adsorption on only one side of the slab was considered. The bottom layer of the slab was fixed in the bulk $\mathrm{Si}$ positions and passivated with two $\mathrm{H}$ atoms per $\mathrm{Si}$ atom. The slabs were electrostatically decoupled along the $c$ direction. All relaxations were considered complete when the forces were less than $10 \mathrm{meV}^{-1}$.

Chemisorption energies are known to be sensitive to the choice of the exchange and correlation (xc) functional. It has been shown that for chemisorbed atoms and molecules on transition metal surfaces, the revPBE functional ${ }^{28}$ generally gives better results than the PBE functional, which tends to overbind the molecules. ${ }^{29}$ A comparison between the present $E_{\text {ads }}$ and those of previous studies is shown in Table I. The PBE and PW91 (Refs. 30 and 31) xc functionals give similar $E_{\text {ads }}$, as expected, but are around two times higher than the revPBE $E_{\text {ads }}$.
The additional binding energy due to vdW forces is calculated using the post-GGA total energy vdW-DF described in Ref. 18. To minimize any artificial exchange binding which can be mistaken from vdW binding, the vdW-DF employs the revPBE form for the exchange description. ${ }^{33,34}$ The vdW-DF replaces the GGA correlation and divides the correlation into a shorter ranged and a longer ranged part. The first part is approximated by the local density approximation, while the latter $\left(E_{\mathrm{nl}}^{c}\right)$ includes the important dispersive interactions. $E_{\mathrm{nl}}^{c}$ is nonlocal by construction and, consistent with the approximation for the shorter ranged correlation, it is constructed to vanish for a homogeneous system. In the vdW-DF, the total energy reads

$$
\begin{aligned}
E^{\mathrm{vdW}-\mathrm{DF}} & =E^{\mathrm{revPBE}}-E_{c}^{\mathrm{revPBE}}+E_{c}^{\mathrm{LDA}}+E_{\mathrm{nl}}^{c} \\
& =E^{\mathrm{vdW} 0}+E_{\mathrm{nl}}^{c},
\end{aligned}
$$

with all terms obtained on the basis of self-consistent semilocal PBE DFT calculations. The nonlocal correlation can be written in the simple form ${ }^{18}$

$$
E_{\mathrm{nl}}^{c}=\frac{1}{2} \int d \mathbf{r} \int d \mathbf{r}^{\prime} n(\mathbf{r}) \phi\left(\mathbf{r}, \mathbf{r}^{\prime}\right) n\left(\mathbf{r}^{\prime}\right),
$$

with a density-density interaction kernel $\phi\left(\mathbf{r}, \mathbf{r}^{\prime}\right)$ derived from the many-body response of the weakly inhomogeneous electron gas. The kernel $\phi\left(\mathbf{r}, \mathbf{r}^{\prime}\right)$ can be tabulated in terms of two dimensionless parameters, a scaled separation $D=\left|\mathbf{r}-\mathbf{r}^{\prime}\right|\left(q_{0}+q_{0}^{\prime}\right) / 2$, and an asymmetry parameter $\delta=\left(q_{0}-q_{0}^{\prime}\right) /\left(q_{0}+q_{0}^{\prime}\right)$, where $q_{0}(\mathbf{r})$ is a local parameter that depends on the electron density and its gradient at $\mathbf{r}$. More information on the explicit form and derivation of the kernel can be found in Ref. 18 .

$E_{\mathrm{nl}}^{c}$ is to be evaluated for a periodic system and we use the scheme developed in Refs. 22 and 35 to evaluate the integral. In short, we let $\mathbf{r}$ in Eq. (2) run through all electron density points within the DFT unit cell. The primed spatial coordinate, on the other hand, includes the electron density from the surrounding lateral repeated images until convergence is obtained. We have found the integral to be converged to include in total five (three) unit cells in each direction for the largest (lowest) coverages.

The post-GGA version of the vdW-DF implemented in this Rapid communication does not allow for any additional geometric and electronic relaxation beyond the PBE determined adsorption structure. Thonhauser et al. ${ }^{21}$ implemented the vdW-DF self-consistently and for noble gas and cytosine dimers, the difference between the self-consistent and nonself-consistent energies was negligible. This study also looked at the effect of the vdW-DF on bulk Si. In this case, as expected, the contribution of the $E_{\mathrm{nl}}^{c}$ energy was negligible and the lattice constant and total energy were similar to those obtained using PBE. This indicates that our results, which use the non-self-consistent implementation, are reliable.

Due to numerical convergence issues of the nonlocal integral, ${ }^{22,23}$ special care is taken when correcting for the nonlocal energy in the adsorption system, leading to additional steps in the evaluation procedure of vdW-DF. First, $E_{\text {ads }}$ between the benzene layer and the surface (BLS) is evaluated, and second, the intralayer molecular-molecular 
TABLE II. vdW-DF $E_{\text {ads }}$ and its contributions are shown for a coverage of 0.5 ML. The standard DFT $\mathrm{PBE}$ and revPBE results are shown for comparison. All energies are in $\mathrm{eV}$.

\begin{tabular}{lrcccc}
\hline \hline & PBE & revPBE & vdW-DF & $E^{\text {vdW0 }}$ & $E_{\text {nl }}^{c}$ \\
\hline BLS (BF) & 3.51 & 3.08 & 3.32 & 1.96 & 1.36 \\
BLS (TB) & 8.51 & 8.04 & 8.02 & 6.56 & 1.46 \\
IMM (BF) & -0.00 & -0.01 & 0.02 & -0.04 & 0.06 \\
IMM (TB) & -0.03 & -0.04 & -0.02 & -0.07 & 0.05 \\
UF (BF) & -2.52 & -2.51 & $(-2.52)$ & & \\
UF (TB) & -7.23 & -7.25 & $(-7.23)$ & & \\
$E_{\text {ads }}(\mathrm{BF})$ & 0.99 & 0.57 & 0.82 & & \\
$E_{\text {ads }}(\mathrm{TB})$ & 1.24 & 0.75 & 0.77 & & \\
\hline \hline
\end{tabular}

(IMM) energy associated by isolating the benzene from the benzene layer is calculated. To ensure maximal error cancellation in the evaluation of the above intermediates, the isolated layer, the silicon surface, and the benzene molecule are fixed in the PBE adsorbant atomic configurations and the spatial separation of the density fast-Fourier transform grid is kept constant in all calculations. Finally, the contribution of unfolding (UF) the isolated molecule and the surface to their PBE relaxed structures is calculated. We choose in accord with Ref. 23 to describe this final energetic contribution within the PBE approximation. This is justified, as all structural information has been obtained self-consistently within the PBE functional, and furthermore, PBE is, in contrary to vdW-DF, designed with the energetics of the internal atomic binding in mind.

The detailed data for the $0.5 \mathrm{ML}$ case are given in Table II, with the vdW-DF total energy separated into the nonlocal correlation $E_{\mathrm{nl}}^{c}$ including dispersion forces and the remaining $E^{\mathrm{vdW} 0}$ part. We will briefly discuss both of the vdW-DF contributions that contain nonlocal components (BLS and IMM) for the $0.5 \mathrm{ML}$ case and compare these to the corresponding $\mathrm{PBE}$ and revPBE results.

For BLS interactions, $E_{\mathrm{nl}}^{c}$ for the TB case is found to be $0.1 \mathrm{eV}$ more attractive than in the BF case. This stems from the fact that the TB structure is more closely bound to the $\mathrm{Si}$ surface, and accordingly, we find $\approx 0.1 \mathrm{eV}$ difference for all coverages. However, $E_{\mathrm{nl}}^{c}$ must be added to $E^{\mathrm{vdW} 0}$ to find the vdW-DF energy. The $E^{\mathrm{vdW} 0}$ of the TB configuration is at first glance considerably larger in the BF case. However, comparing the combined vdW-DF energy with the corresponding revPBE value, we see that the TB case has almost the same energy, while the $\mathrm{BF}$ case is about $0.24 \mathrm{eV}$ more attractive. Thus, compared to the revPBE correlation energy, the BLS part of the vdW-DF correlation increases the BF $E_{\text {ads }}$ by $0.26 \mathrm{eV}$ more than in the TB case. This is in accord with the expectation that the BF structure has a larger vdW-like interaction than the more covalently bound TB structure.

For IMM interactions, we find $E_{\mathrm{nl}}^{c}$ to be small and attractive $(\approx 0.05 \mathrm{eV})$ while $E^{\mathrm{vdW} 0}$ is slightly repulsive in both the $\mathrm{TB}$ and $\mathrm{BF}$ configurations. The IMM $E^{\mathrm{vdW} 0}$ is found to be slightly more repulsive for the TB configuration than for the $\mathrm{BF}$, such that the net vdW-DF energy is slightly repulsive for the TB case and weakly attractive for the BF case, closely resembling the interactions obtained in the semilocal revPBE and PBE approximations. As the coverage is reduced, the vdW-DF IMM interactions are even less pronounced and can be described with the semilocal DFT functionals to within $0.02 \mathrm{eV}$.

Overall, the change in $E_{\text {ads }}$ calculated with vdW-DF is substantial, in particular, for the BF structure. The main contribution to $E_{\mathrm{ads}}$ comes from $E_{\mathrm{nl}}^{c}$ between the surface and the benzene layer. The attraction between the benzene molecules in the layer is minimal. This behavior is similar to that of a cytosine dimer ${ }^{21}$ where the repulsive $E^{\mathrm{vdW} 0}$ term is compensated by the attractive $E_{\mathrm{nl}}^{c}$ to give an overall binding energy of around $0.3 \mathrm{eV}$.

We will now discuss the effect of coverage on the adsorption energy. The adsorption energies for various coverages are shown in Table III. Both PBE and revPBE favor the TB configuration and, as expected, PBE predicts the larger adsorption energy. On the contrary, vdW-DF, with its account of the dispersion interactions, predicts the BF configuration to be slightly favored. For a coverage of $0.25 \mathrm{ML}$, two supercell orientations are possible and are denoted $\mathrm{a}$ and b with primitive lattice vectors $(220)(\overline{1} 10)(006)$ and $(110)(\overline{2} 20)(006)$, respectively. In general, only a minimal coverage dependence is found as changes in $E_{\mathrm{nl}}^{c}$ are almost canceled by the corresponding changes in $E^{\mathrm{vdW} 0}$. Correspondingly, the vdW-DF adsorption energies in the BF case only show a small increase with increasing coverage. For the TB structure, the coverage dependence is almost constant

TABLE III. Variation of adsorption energy with coverage for the $\mathrm{BF}$ and TB structures. Both the PBE and vdW-DF results are shown for comparison. All energies are in $\mathrm{eV}$.

\begin{tabular}{lccc}
\hline \hline & PBE & revPBE & vdW-DF \\
\hline BF-0.5 & 0.99 & 0.57 & 0.82 \\
BF-0.25a & 1.02 & 0.59 & 0.82 \\
BF-0.25b & 1.02 & 0.60 & 0.84 \\
BF-0.125 & 1.04 & 0.62 & 0.84 \\
TB-0.5 & 1.24 & 0.75 & 0.77 \\
TB-0.25a & 1.23 & 0.74 & 0.74 \\
TB-0.25b & 1.33 & 0.84 & 0.86 \\
TB-0.125 & 1.31 & 0.81 & 0.82 \\
\hline \hline
\end{tabular}


except for the $0.25 \mathrm{~b}$ coverage, which has a pronounced preference. This effect is also observed for the semilocal functionals. For all coverages, the semilocal functionals favor the $\mathrm{TB}$ configuration. In contrast, vdW-DF predicts that the BF structure is stable for all cases, except $0.25 \mathrm{~b}$, where the TB is lower in energy by only $0.02 \mathrm{eV}$.

In the $0.125 \mathrm{ML}$ case, the nonlocal IMM interactions are less than $0.01 \mathrm{eV}$, so the monomers can be regarded as isolated. The difference between the $\mathrm{BF}$ and $\mathrm{TB}$ configurations is thus $0.02 \mathrm{eV}$ in the isolated case, which is slightly less than the $0.08 \mathrm{eV}$ difference found in a corresponding MP2 calculation. ${ }^{17}$ To compare with experimental data, we used the Redhead equation ${ }^{36}$ to estimate the adsorption energies based on thermal desorption spectra in Ref. 8. For a peak temperature of $432 \mathrm{~K}$, a heating rate of $5 \mathrm{~K} \mathrm{~s}^{-1}$, and a preexponential frequency factor between $10^{12}$ and $10^{16} \mathrm{~s}^{-1}$, we obtain an adsorption energy in the range 1.06-1.40 eV. The difference between experiment and vdW-DF energies is due to the uncertainty in the GGA exchange energies, ${ }^{19}$ which can be seen in Table II. The use of PBE with the vdW-DF increases the adsorption energies to within the experimental range but the uncertainty in the exchange energy is large enough to mask the small energy differences between the two structures, particularly for the lower coverages.

In summary, we have demonstrated that standard DFT adsorption energies are significantly dependent on the $\mathrm{xc}$ functional and, furthermore, that the inclusion of $\mathrm{vdW}$ forces makes a qualitative difference to the results. Standard DFT with $\mathrm{PBE}$ and revPBE functionals finds that the TB structure is always stable, whereas vdW-DF DFT calculations find that, for some coverages, vdW forces stabilize the BF structure. These results have significant implications for many DFT studies as vdW forces are generally considered to have a negligible effect on covalently bonded systems and are usually ignored.

K.J. would like to thank Andris Gulans for valuable discussions. This work was supported by the Finnish Funding Agency for Technology and Innovation (TEKES) and the Academy of Finland (Center of Excellence 2006-2011). Computational resources were provided by the Center for Scientific Computing (CSC), Finland. The Lundbeck Foundation (Denmark) is gratefully acknowledged.
${ }^{1}$ M. C. Hersam, N. P. Guisinger, and J. W. Lyding, Nanotechnology 11, 70 (2000).

${ }^{2}$ S. F. Bent, Surf. Sci. 500, 879 (2002).

${ }^{3}$ A.-S. Duwez, S. Cuenot, C. Jérôme, S. Gabriel, R. Jérôme, S. Rapino, and F. Zerbetto, Nat. Nanotechnol. 1, 122 (2006).

${ }^{4}$ I. Zanella, A. Fazzio, and A. J. R. da Silva, J. Phys. Chem. B 110, 10849 (2006).

${ }^{5}$ S. Y. Quek, J. B. Neaton, M. S. Hybertsen, E. Kaxiras, and S. G. Louie, Phys. Rev. Lett. 98, 066807 (2007).

${ }^{6}$ I. Štich, M. C. Payne, R. D. King-Smith, J.-S. Lin, and L. J. Clarke, Phys. Rev. Lett. 68, 1351 (1992).

${ }^{7}$ V. R. Cooper, T. Thonhauser, A. Puzder, E. Schröder, B. I. Lundqvist, and D. C. Langreth, J. Am. Chem. Soc. 130, 1304 (2007).

${ }^{8}$ S. Gokhale, P. Trischberger, D. Menzel, W. Widdra, H. Dröge, H. P. Steinrück, U. Birkenheuer, U. Gutdeutsch, and N. Rösch, J. Chem. Phys. 108, 5554 (1998).

${ }^{9}$ N. Witkowski, F. Hennies, A. Pietzsch, S. Mattsson, A. Föhlisch, W. Wurth, M. Nagasono, and M. N. Piancastelli, Phys. Rev. B 68, 115408 (2003).

${ }^{10}$ N. Witkowski, O. Pluchery, and Y. Borensztein, Phys. Rev. B 72, 075354 (2005).

${ }^{11}$ G. P. Lopinski, D. J. Moffatt, and R. A. Wolkow, Chem. Phys. Lett. 282, 305 (1998).

${ }^{12}$ G. P. Lopinski, T. M. Fortier, D. J. Moffatt, and R. A. Wolkow, J. Vac. Sci. Technol. A 16, 1037 (1998).

${ }^{13}$ Y. K. Kim, M. H. Lee, and H. W. Yeom, Phys. Rev. B 71, 115311 (2005).

${ }^{14}$ W. A. Hofer, A. J. Fisher, G. P. Lopinski, and R. A. Wolkow, Phys. Rev. B 63, 085314 (2001).

${ }^{15}$ J.-Y. Lee and J.-H. Cho, Phys. Rev. B 72, 235317 (2005).

${ }^{16}$ M. Mamatkulov, L. Stauffer, C. Minot, and P. Sonnet, Phys. Rev. B 73, 035321 (2006).

${ }^{17}$ Y. Jung and M. S. Gordon, J. Am. Chem. Soc. 127, 3131 (2005).

${ }^{18}$ M. Dion, H. Rydberg, E. Schröder, D. C. Langreth, and B. I. Lundqvist, Phys. Rev. Lett. 92, 246401 (2004).

${ }^{19}$ A. Puzder, M. Dion, and D. C. Langreth, J. Chem. Phys. 124,
164105 (2006).

${ }^{20}$ T. Thonhauser, A. Puzder, and D. C. Langreth, J. Chem. Phys. 124, 164106 (2006).

${ }^{21}$ T. Thonhauser, V. R. Cooper, S. Li, A. Puzder, P. Hyldgaard, and D. C. Langreth, Phys. Rev. B 76, 125112 (2007).

${ }^{22}$ E. Ziambaras, J. Kleis, E. Schröder, and P. Hyldgaard, Phys. Rev. B 76, 155425 (2007).

${ }^{23}$ S. D. Chakarova-Käck, E. Schröder, B. I. Lundqvist, and D. C. Langreth, Phys. Rev. Lett. 96, 146107 (2006).

${ }^{24}$ Open source plane-wave DFT code DACAPO (http:// www.fysik.dtu.dk/CAMPOS/).

${ }^{25}$ J. P. Perdew, K. Burke, and M. Ernzerhof, Phys. Rev. Lett. 77, 3865 (1996).

${ }^{26}$ J. P. Perdew, K. Burke, and M. Ernzerhof, Phys. Rev. Lett. 78, 1396(E) (1997).

${ }^{27}$ J. P. Perdew, K. Burke, A. Zupan, and M. Ernzerhof, J. Chem. Phys. 108, 1522 (1998).

${ }^{28}$ Y. Zhang and W. Yang, Phys. Rev. Lett. 80, 890 (1998).

${ }^{29}$ B. Hammer, L. B. Hansen, and J. K. Norskov, Phys. Rev. B 59, 7413 (1999).

${ }^{30}$ J. P. Perdew, Electronic Structure of Solids '91 (Akademie Verlag, Berlin, 1991), p. 11.

${ }^{31}$ J. P. Perdew, K. Burke, and Y. Wang, Phys. Rev. B 54, 16533 (1996).

${ }^{32}$ K. Johnston and R. M. Nieminen, Phys. Rev. B 76, 085402 (2007).

${ }^{33}$ X. Wu, M. C. Vargas, S. Nayak, V. Lotrich, and G. Scoles, J. Chem. Phys. 115, 8748 (2001).

${ }^{34}$ D. C. Langreth, M. Dion, H. Rydberg, E. Schröder, P. Hyldgaard, and B. I. Lundqvist, Int. J. Quantum Chem. 101, 599 (2005).

${ }^{35}$ J. Kleis, B. I. Lundqvist, D. C. Langreth, and E. Schröder, Phys. Rev. B 76, 100201(R) (2007).

${ }^{36}$ P. A. Redhead, Vacuum 12, 203 (1962).

${ }^{37} \mathrm{In}$ this paper, $1 \mathrm{ML}$ corresponds to one benzene molecule per $\mathrm{Si}$ dimer. 